Ophthalmologe 2013 · 110:470

DOI 10.1007/s00347-013-2865-3

Online publiziert: 15 Mai 2013

c) Springer-Verlag Berlin Heidelberg 2013

\title{
Transplantatversagen immunologisch begründet?
}

\section{Erwiderung}

\section{S. Schmidt, M.P. Holzer, R. Khoramnia, G.U. Auffarth}

International Vision Correction Research Centre, Universitäts-Augenklinik, Heidelberg

\section{Leserbrief}

\section{Lange, D. Böhringer, T. Reinhard \\ Universitäts-Augenklinik, Freiburg}

Mit Interesse haben wir die Kasuistik von Schmidt et al. gelesen, in der eine „Abstoßungsreaktion“ eines vom Partnerauge stammenden (autologen) Hornhauttransplantats 30 Monate nach Keratoplastik beschrieben wird. Eine immunologische Abstoßungsreaktion gegen autologes Material kann es definitionsgemäß nicht geben. Die Autoren beschreiben vielmehr ein immunologisches (= entzündliches) Transplantatversagen, das beispielsweise im Rahmen einer Infektion aufgetreten sein könnte. In Anbetracht des äußerst ungünstigen Verlaufs mit insgesamt 6 weiteren Transplantatversagen und der hoch dosierten Immunsupression, kommt insbesondere eine herpetische Grunderkrankung infrage.

\section{Korrespondierender Autor}

Dr. C. Lange, M.D., Ph.D.

Universitäts-Augenklinik

Killianstr. 5

79106 Freiburg

clemenslange@hotmail.com
Sehr geehrter Herr Lange,

wir bedanken uns für Ihren Leserbrief zum Fallbericht „Immunologisches Transplantatversagen nach autologer kontralateraler Keratoplastik“.

Es ist nachvollziehbar, dass Sie eine immunologische Abstoßungsreaktion im beschriebenen Fall infrage stellen und eine infektiös-entzündliche Genese vermuten.

Im Fall wurde beschrieben, wie zu Beginn der initialen Abstoßungsreaktion eine intensivierte antientzündliche und antiangiogenetische Therapie eingeleitet und über mehrere Wochen fortgeführt wurde. Die Therapie beinhaltete sowohl eine hoch dosierte antibiotische als auch antivirale Medikation in lokaler und systemischer Applikation. Von der Durchführung einer Vorderkammerpunktion zum möglichen Nachweis einer herpetischen Genese wurde aufgrund des klinischen Gesamtbildes abgesehen. Die These einer infektiösen Reaktion kann somit nicht labormedizinisch widerlegt werden, erscheint jedoch aufgrund der beschriebenen Behandlungsschemata aus unserer Sicht unwahrscheinlich.

\section{Korrespondierender Autor}

\section{Dr. S. Schmidt}

International Vision Correction Research Centre Universitäts-Augenklinik

Im Neuenheimer Feld 400

69120 Heidelberg

stephan_schmidt307@yahoo.de

\section{Erwiderung}

\section{Lange, D. Böhringer, T. Reinhard}

Universitäts-Augenklinik, Freiburg

Sehr geehrter Herr Schmidt, vielen Dank für Ihre Zusatzinformation, dass in dem Kasus initial eine antivirale Therapie begonnen wurde. Wir stimmen Ihnen zu, dass eine Vorderkammerpunktion zur Herpesdiagnostik aufgrund der geringen Sensitivität nicht zielführend ist. Das Nicht-Ansprechen einer antiviralen Therapie, die in der ursprünglichen Kasuistik im Übrigen nicht erwähnt wird, schließt eine Herpesinfektion jedoch ebenso wenig aus.

\section{Korrespondierender Autor}

Dr. C. Lange, M.D., Ph.D.

Universitäts-Augenklinik

Killianstr. 5

79106 Freiburg

clemenslange@hotmail.com

Interessenkonflikt. Die korrespondierenden Autoren geben für sich und ihre Koautoren an, dass kein Interessenkonflikt besteht. 